\title{
On the Capacity of Cognitive Radio Sensor Networks: From Physical Layer to Network Layer
}

\author{
Ting Dong \\ Department Of Information Engineering, Yulin University, Shanxi Yulin, 719000, \\ China \\ dongtingwu@hotmail.com
}

\begin{abstract}
Cognitive Radio has stirred great interest recently, with its tremendous potential to further exploit the scarce spectrum resource. Among the diverse research topics, the fundamental capacity issue deserves a thorough study since it serves as a principle for future implementation and performance evaluation. Existing researches on cognitive capacity mainly fall into two categories: channel capacity and network capacity. The former can accurately portray the pairwise cognitive channel capacity, but less address the case with multiple secondary users. The latter investigates the throughput of largescale network with respect to its size and topology, while ignoring crucial factors in physical layer. Recognizing their strengths and limitations, this paper aims to present a more comprehensive view of the cognitive capacity issue. First, we extend the information theoretic model to a special case in multi-user scenario, which well characterizes the capacity of cognitive multiple access channel (MAC). Second, we analyze network scaling by incorporating sensing errors, and show that physical layer features may affect the throughput performance. Last but not least, our analysis not only extends the existing researches but also sheds some light on the cross-layer study of cognitive capacity.
\end{abstract}

Keywords: Cognitive Radio, Interference Channel, Side Information, Multi-User Capacity, Cross-Layer Analysis, Scaling Law

\section{Introduction}

With the explosive growth of data traffic, the fixed spectrum allocation policy can no longer meet the exponentially increasing bandwidth demand. However, we observe that the wireless resource is still underutilized in terms of spectrum occupancy[1].Therefore, designing a network paradigm to utilize the spectrum in a more efficient manner has become an urgent issue. Cognitive radio is such a paradigm that allows the cognitive user to dynamically access the spectrum as long as it can avoid harmful interference with licensed or unlicensed users [2]. Till now, various research fields have been developed to analyze cognitive systems, such as biomedical area [3-4], image processing [5-6, 26-28], and wireless network [7-8, 23-24]. Among them, the fundamental study of capacity issue aims to reveal the limit of performance, and guide us to the optimal system design.

Current researches on cognitive capacity mainly fall into two categories: channel capacity and network capacity. The former is based on modeling various kinds of information theoretic cognitive channels. In underlay approach, the cognitive signal is treated as interference to the primary receiver, and simultaneous transmissions of primary users and secondary users are allowed. Assuming that the channel gain between cognitive transmitter and non-cognitive receiver is known by the former, the mutual interference can be restricted within an acceptable range. Though easy to implement, the underlay approach only supports low-rate communication due to its strict power restriction. An alternative overlay approach is proposed to increase the transmission rate, where the 
cognitive channel is also known as "interference channel with degraded message sets (ICDMS)". The main idea is to exploit the knowledge of primary signal to cancel or mitigate mutual interference, thus increase the capacity of both primary and secondary systems. While the above approaches permit unharmful interference in nature, most practical cognitive systems seek to avoid interference [24]. Specifically, the secondary transmitter first senses the spectrum, and transmits on the temporarily vacant band. In these cases, the interference channel model no longer applies and we have to view the primary user activity (idle or busy) as side information to the secondary users. While the existing information theoretic paradigm models the physical layer in detail, few of them consider the multi-user scenario. Hence, we derive the capacity region of a cognitive MAC channel, which is a special case that well characterizes information aggregation process in Cognitive Radio Sensor Networks.

A major drawback of scaling law study is that they ignore many physical layer features, such as spectrum sensing, modulation. Therefore, we reconsider the throughput scaling of primary and secondary networks with physical layer sensing error. Through such a joint physical layer - network layer perspective, we discover that the existing scaling results may be degraded in some cases.

\section{Primary Activity as Side Information}

The above mentioned underlay and overlay scenarios permit concurrent transmission of non-cognitive and cognitive radio, as long as the performance of both systems does not degrade. Alternatively, many existing cognitive systems seek to completely avoid the undesired interference by only accessing the spectral holes. The spectral hole is the spectrum resource in time and frequency domain that is temporarily not occupied by primary users.

\subsection{Two-Switch Cognitive Channel Model}

The two-switch cognitive channel model [9] was first proposed by S. Jafar, and it is based on the results of channel capacity with causal/non-causal side information at the transmitter/receiver.

In Figure 1, both the cognitive transmitter (CT) and the cognitive receiver (CR) can sense the PU activity within a sensing region. CT and CR have two switches to control the transmission and receiving of secondary signals. Each of them will stop transmitting/receiving if it detects any primary transmission. Since the PU is either active or inactive, the states of switch at CT and CR are either 1 or 0 . With a power constraint $\mathrm{P}$ for CT, the two-switch cognitive channel model [9] can be formulated as follows:

$$
Y=\left(S_{T} X+Z\right) S_{R}
$$

where, $S_{T}, S_{R} \in\{0,1\}, \mathrm{E}\left[|X|^{2} S_{T}\right] \leq P$.

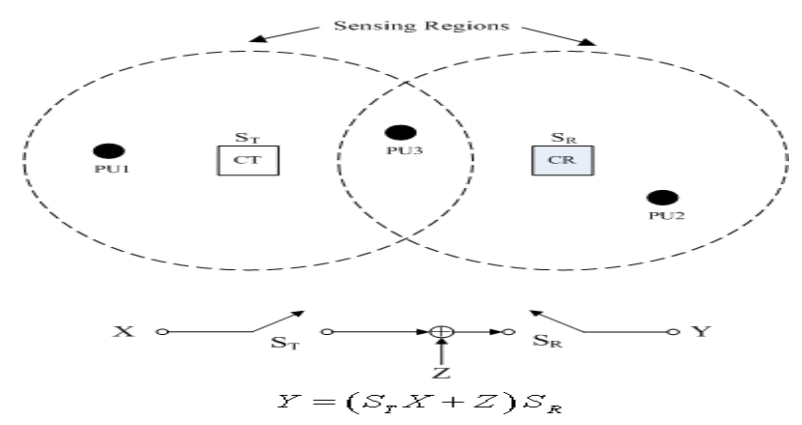

Figure 1. Two-Switch Cognitive Channel 
Recall the existing results in channel capacity with side information [9]:

A. channel capacity with non-causal side information at both the transmitter and receiver $S_{T} \rightarrow U \rightarrow X$ forms a Markov chain $C_{S_{T}, S_{R}}^{\text {non-causal }}=\max _{P} I\left(U ; Y, S_{R}\right)-I\left(U ; S_{T}\right)$

$$
P_{\text {non-causal }}=\left\{p\left(U ; X \mid S_{T}\right)=p\left(U \mid S_{T}\right) p\left(X \mid U, S_{T}\right)\right\}
$$

B. channel capacity with causal side information at both the transmitter and receiver

$$
\begin{aligned}
& \left.\begin{array}{l}
S_{T} \\
U
\end{array}\right\} \rightarrow X \\
& C_{S_{T}, S_{R}}^{\text {causal }}=\max _{P_{\text {causal }}} I\left(U ; Y, S_{R}\right)-I\left(U ; S_{T}\right) \\
& P_{\text {causal }}=\left\{p\left(U ; X \mid S_{T}\right)=p(U) p\left(X \mid U, S_{T}\right)\right\}
\end{aligned}
$$

Based on the above results, the following theorems are proved by S. Jafar [9]:

Theorem 1: For the two switch channel with non-causal side information at the transmitter, coding can be performed directly on the input alphabet (i.e., $\mathrm{U}=\mathrm{X}$ ) and the channel capacity is:

$$
C_{S_{T}, S_{R}}^{\text {non-causal }}=\max _{p\left(X \mid S_{T}\right)} I\left(X ; Y, S_{R}\right)-I\left(X ; S_{T}\right)
$$

Theorem 2: For the two switch channel with causal side information at the transmitter, coding can be performed directly on the input alphabet (i.e., $\mathrm{U}=\mathrm{X}$ ) and the channel capacity is:

$$
C_{S_{T}, S_{R}}^{\text {causal }}=\max _{p(X)} I\left(X ; Y, S_{R}\right)
$$

\subsection{Involving More Nodes: Multi-User Scenario}

While the capacity of single cognitive transmitter-receiver pair has been well investigated, we attempt to extend it to the scenario with more nodes involved. A very typical setting is the Cognitive Radio Sensor Network (CRSN) [10-12], where nodes are grouped into clusters according to their location and common channels. The cluster members $(\mathrm{CM})$ report the sensed information to cluster head $(\mathrm{CH})$ through multiple access channel (MAC) in uplink. This clustered topology greatly facilitates the convergence of collected information. Therefore, it makes sense to explore the capacity of cognitive MAC channel with side information.

The cognitive MAC channel with side information (primary activity) is illustrated in Figure 2: 


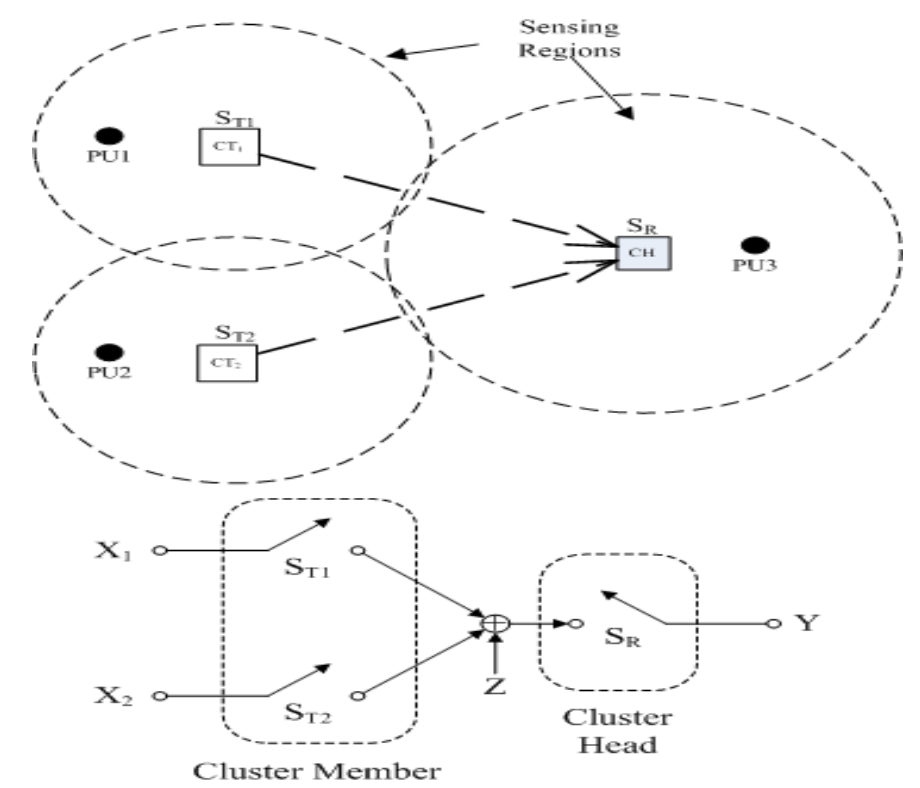

Figure 2. Cognitive MAC Channel

As shown in the Figure 2, both the two cognitive transmitters (CT1, CT2) and the cognitive receiver $(\mathrm{CH})$ can sense the PU activity within a sensing region. Each of them will stop transmitting/receiving if it detects any primary transmission. The states of switch at $\mathrm{CT} 1,2$ and $\mathrm{CH}$ are either 1 or 0 . With power constraints for the transmitters, the cognitive MAC channel model can be formulated as follows:

$$
Y=\left(S_{T_{1}} X_{1}+S_{T_{2}} X_{2}+Z\right) S_{R}
$$

where $S_{T_{1}}, S_{T_{2}}, S_{R} \in\{0,1\}, \quad \mathrm{E}\left[\left|X_{1}\right|^{2} S_{T_{1}}\right] \leq P_{1}, \quad \mathrm{E}\left[\left|X_{2}\right|^{2} S_{T_{2}}\right] \leq P_{2}$. For convenience, unit power of noise is assumed: $\mathrm{E}\left[|Z|^{2}\right]=1$.

Theorem 3: For the cognitive MAC channel with causal side information $S_{T_{1}}, S_{T_{2}}$ at the transmitters and side information $S_{R}$ at the receiver, coding can be performed directly on the input alphabets (i.e., $U_{1}=X_{1}, U_{2}=X_{2}$ ) and the channel capacity region is:

$$
C_{S_{T_{1}}, S_{T_{2}}, S_{R}}^{\text {causal }}=\left\{\begin{array}{c}
R_{1}=\max _{p\left(X_{1}\right) p\left(X_{2}\right)} I\left(X_{1} ; Y, S_{R} \mid X_{2}\right) \\
R_{2}=\max _{p\left(X_{1}\right) p\left(X_{2}\right)} I\left(X_{2} ; Y, S_{R} \mid X_{1}\right) \\
R_{1}+R_{2}=\max _{p\left(X_{1}\right) p\left(X_{2}\right)} I\left(X_{1}, X_{2} ; Y, S_{R}\right)
\end{array}\right.
$$

\section{Capacity Scaling: A Network Layer View}

\subsection{Scaling Laws Of Cognitive Networks}

The exploring of large-scale network capacity was initiated by Gupta and Kumar [13], which focus on the relationship between network throughput scaling and the network size. For cognitive networks, the main results in [14] [15] is that as long as the secondary user is denser than primary user in the order sense, the primary and secondary network can achieve scaling as two standalone networks: 
$\Theta\left(\sqrt{\frac{1}{n \log n}}\right)$ and $\Theta\left(\sqrt{\frac{1}{m \log m}}\right)$ respectively. Recently, an intelligent design of highway system is introduced in [16], in which both networks are tessellated into cells. There is either a vertical edge or a horizontal edge in each cell, see Figure 4. A fraction of the edges form vertical and horizontal relay paths, acting as the backbones for information dissemination. With this relaying structure, the throughput of both networks can be improved to $\Theta\left(\frac{1}{\sqrt{n}}\right)$ and $\Theta\left(\frac{1}{\sqrt{m}}\right)$ [17], and this result is the best known till now, and is widely accepted.

\subsection{The Price Paid For Accurate Sensing}

Considering energy efficient cognitive radio system, most of existing papers only take the transmitting power into account. The power consumption in spectrum sensing has not been paid enough attention to. However, the power consumption in radio listening for sensor nodes should not be neglected. It is shown in [18] that the power consumptions in sensing and transmitting are actually in the same order. Specifically, in Software Radio, the spectrum sensing power is mainly consumed in the process of high frequency A/D converting. According to [19], the increase in sampling rate leads to a proportionate increase in power consumption. Therefore, when utilizing energy detection in spectrum sensing, we have to pay more power to achieve better detection performance.

The tradeoff between energy consumption and detection performance in spectrum sensing is briefly introduced below. We use the probability of miss detection and the probability of false alarm as the performance metric for sensing accuracy.

The discrete signal at the cognitive receiver can be represented as:

$$
\begin{aligned}
& y(n)=s(n)+u(n) \rightarrow \mathrm{H}_{1}: \mathrm{PU} \text { Active } \\
& y(n)=u(n) \rightarrow \mathrm{H}_{0}: \mathrm{PU} \text { Inactive } \\
& E\left[|s(n)|^{2}\right]=\sigma_{s}^{2}, \quad E\left[|u(n)|^{2}\right]=\sigma_{u}^{2}
\end{aligned}
$$

The $N$ point energy detector is as follows:

$$
T(y)=\frac{1}{N} \sum_{n=1}^{N}|y(n)|^{2}
$$

The corresponding false alarm and miss detection probability are [20]:

$$
\begin{gathered}
P_{f}(\varepsilon, N)=Q\left(\left(\frac{\varepsilon}{\sigma_{u}^{2}}-1\right) \sqrt{N}\right) \\
P_{m}(\varepsilon, N)=1-Q\left(\left(\frac{\varepsilon-\sigma_{s}^{2}}{\sigma_{u}^{2}}-1\right) \sqrt{\frac{N}{2 \sigma_{s}^{2} / \sigma_{u}^{2}+1}}\right)
\end{gathered}
$$

where $Q(x)=\frac{1}{2 \pi} \int_{x}^{\infty} \exp \left(-\frac{t^{2}}{2}\right) d t$ is the Q function, and $\varepsilon$ is the detection threshold.

Assuming $E_{\text {sample }}$ to be the per sample energy, we derive the energy consumption of spectrum sensing as a function of miss-detection probability $P_{m}$ and false alarm probability $P_{f}$ : 


$$
E_{\text {sensing }}=\left(\frac{Q^{-1}\left(P_{f}\right)-\sqrt{2 \sigma_{s}^{2} / \sigma_{u}^{2}+1} Q^{-1}\left(1-P_{m}\right)}{\sigma_{s}^{2} / \sigma_{u}^{2}}\right)^{2} E_{\text {sample }}
$$

As illustrated in Figure 6, we observe that the lower sensing error $\left(P_{m}\right.$ or $\left.P_{f}\right)$ directly leads to higher sensing energy according to the simulation result.

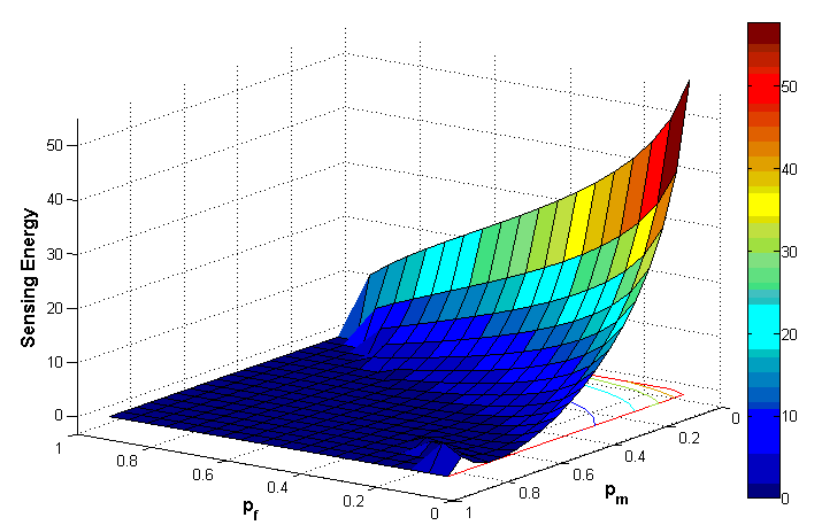

Figure 3. Energy Paid for Accurate Spectrum Sensing

\subsection{A Joint Physical Layer-Network Layer Perspective}

As we know, all existing works on the scaling law of cognitive networks [21] etc., assume perfect spectrum sensing, i.e., the exact state of primary transmitters and corresponding preservation regions are known by secondary system, free of error. However, from the viewpoint of physical layer, the energy and hardware resources we pay for accurate sensing cannot be neglected. As described in the previous subsection, we can only obtain sensing results bounded by certain error level with limited resources.

If take into account these physical layer features (different levels of sensing errors), will the throughput scaling of the primary network and secondary networks be severely degraded or not affected at all? In this subsection, we attempt to present some preliminary results.

From [22] introducing the highway system based on the percolation theory, we say the edge is open if there is at least one node inside the cell. If the nodes are deployed according to a Poisson Point Process with unit density, the edge open rate is: $p \equiv 1-e^{-c^{2}}$, where ${ }_{c}$ is the side length of the cell. Evolving into the cognitive scenario, the edge is open if both: 1 . At least one secondary node in the cell. 2. No primary users in either this cell or its 8 neighboring cells. Therefore, the edge open rate for secondary network is

$$
p \equiv p_{1} p_{2}=\left(1-e^{-c_{s}^{2}}\right) e^{-\frac{9 n}{m} c_{s}^{2}}
$$

When the active primary user is correctly detected, the neighboring area is claimed as preservation region, which is represented in red in Figure 4. Now we consider the two types of sensing errors also shown in Figure 4: 


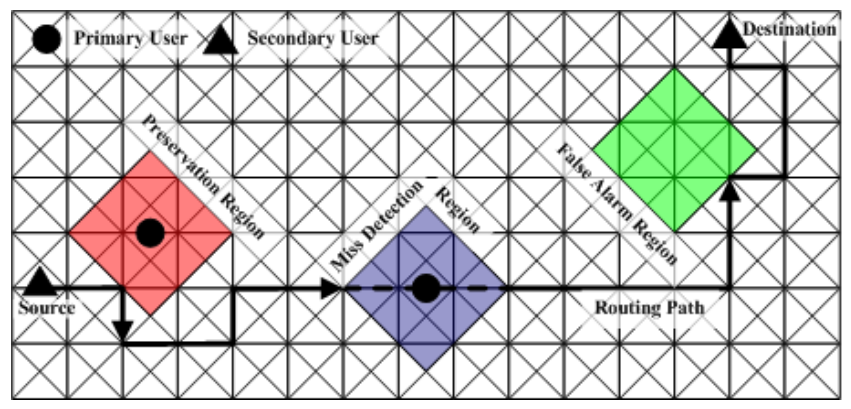

\section{Figure 4. Sensing Errors in Overlaid Networks}

In the case of miss detection with probability $p_{m}$ (range from 0 to 1 ), a cell (edge) will be regarded as open, while it is actually in the preservation region (represented in blue as miss detection region). The scheduler will guide a highway through this cell, and a collision may occur, both the primary and secondary receiver will fail to decode.

In the case of false alarm with probability $p_{f}$ (range from 0 to 1 ), a cell (edge) will be regarded as closed, while it is actually outside the preservation region (represented in green as false alarm region). The scheduler will keep the highway away from this cell, therefore the open edge resource is wasted, and the probability that the scheduler claim a secondary cell open is reduced.

With sensing error, the primary cell is open with probability:

$$
p_{p} \equiv\left(1-e^{-c_{s}^{2}}\right)\left(1-p_{m}\right)
$$

The secondary cell is open with probability:

$$
p_{s} \equiv\left(1-e^{-c_{s}^{2}}\right) e^{-\frac{9 n}{m} c_{s}^{2}}\left(1-p_{f}\right)
$$

From the percolation theory, the threshold for percolation will be affected. In the following, we show this physical layer feature (sensing error rate) may hinder the establishment of the highway system and even modify some of the properties in throughput scaling.

In the highway system, an edge open rate larger than $\frac{5}{6}$ is required to ensure the $\Theta\left(\frac{1}{\sqrt{n}}\right)$ scaling law, see Theorem 5 in [12]. Incorporating sensing error, if $p_{m}$ and $p_{f}$ are less than $\frac{1}{6}$, we may still construct proper tessellation to maintain the performance in terms of scaling, only that the delay would be increased due to packet collision and rerouting. However, if $p_{m}$ and $p_{f}$ are larger than $\frac{1}{6}$, the edge open rate will be less than $\frac{5}{6}$ even if the cell's side length $c_{s}$ goes to infinity. According to the percolation theory, the highway system cannot be constructed due to insufficient number of highways, and $\Theta\left(\frac{1}{\sqrt{n}}\right)$ scaling may be degraded.

\section{Conclusions and Future Works}

In this paper, we present a comprehensive view of the capacity issue in cognitive systems. In Section 2, we use the interference channel to model the cognitive capacity, in 
which both underlay and overlay approaches are discussed. In section 3, we analyze the capacity by taking the primary activity as side information, and extend the information theoretic model to a special case in multi-user scenario that characterizes the capacity of cognitive MAC channel. In section 4, we analyze the throughput scaling of cognitive network by incorporating sensing errors, and showed that physical layer features may affect the overall throughput performance. On the one hand, our analysis extends the existing cognitive capacity researches and provides more general understandings of the topic. On the other hand, we find that the physical layer and network layer should not be separated in capacity analysis. A cross-layer perspective would reveal more interesting results and moves the theoretical study one step forward to practical implementation. From our viewpoint, the existing works are still far from sufficient to push forward the popularization of cognitive radio industry. As for future works, a more unified theoretical system is required, in order to characterize more sophisticated cognitive systems. Moreover, the researchers should include more practical factors, such as coding schemes and network mobility, etc., and see how they change the characteristics of system performance

\section{References}

[1] M. A. McHenry, "NSF Spectrum Occupancy Measurements Project Summary", In Shared Spectrum Company Report, (2005) August.

[2] S. Haykin, "Cognitive Radio: Brain-Empowered Wireless Communications", IEEE Journal on Selected Areas in Communications, vol. 23, (2005), pp. 201-220.

[3] Y. Wang, "A Conceptual Cellular Interaction Model of Left Ventricular Remodelling Post-MI: Dynamic Network with Exit-Entry Competition Strategy”, BMC Systems Biology, vol. 4, no. S5, (2010).

[4] Y. Wang, "Mathematical Modeling and Stability Analysis of Macrophage Activation in Left Ventricular Remodeling Post-Myocardial Infarction”, BMC Genomics, vol. 13, no. 6, (2012).

[5] J. Shen and W. Tan, "Image-Based Indoor Place-Finder Using Image to Plane Matching", Multimedia and Expo (ICME). Proceedings of 2013 IEEE International Conference, Kyoto, Japan, (2013) July.

[6] J. Shen and S. Cheung, "Layer Depth Denoising and Completion for Structured-Light RGB-D Cameras", In 2013 IEEE Conference on Computer Vision and Pattern Recognition (CVPR), (2013).

[7] S. Chen, Y. Zhao, M. Peng and Y. Wang, "A Codesigned Compact Dual-Band Filtering Antenna with PIN Loaded for WLAN Applications”, International Journal of Antennas and Propagation, (2014) May.

[8] J. Yang and Z. Fei, "Bipartite Graph Based Dynamic Spectrum Allocation For Wireless Mesh Networks", In 28th International Conference on Distributed Computing Systems Workshops, Beijing, China, (2008) August.

[9] S. A. Jafar and S. Srinivasa, "Capacity Limits of Cognitive Radio with Distributed and Dynamic Spectral Activity", IEEE Journal on Selected Areas in Communications, vol. 25, (2007) April, pp. 529537.

[10] H. Zhang, "Distributed Spectrum-Aware Clustering In Cognitive Radio Sensor Networks", Global Telecommunications Conference (GLOBECOM 2011), Houston, Texas, USA, (2011) December 5-9.

[11] H. Zhang, Z. Zhang and Y. Chau, "Distributed Compressed Wideband Sensing in Cognitive Radio Sensor Networks", Proceedings of 2011 IEEE Computer Communications Workshops (INFOCOM WKSHPS): Guangzhou, China, (2011) July, pp. 231-245.

[12] G. Prasad Joshi, S. Y. Nam and S. W. Kim, "Cognitive Radio Wireless Sensor Networks: Applications, Challenges and Research Trends. Sensors", vol. 13, no. 9, (2013), pp. 11196-11228.

[13] P. Gupta and P. R. Kumar, "The Capacity of Wireless Networks", IEEE Transactions on Information Theory, vol. 42, (2000) March, pp. 388-404.

[14] S. Jeon, N. Devroye, M. Vu, S. Chung and V. Tarokh, "Cognitive Networks Achieve Throughput Scaling of a Homogeneous Network", IEEE Transactions on Information Theory, vol. 20, no. 1, (2009)

[15] C. Yin, L. Gao and S. Cui, "Scaling Laws For Overlaid Wireless Networks: A Cognitive Radio Network Vs. A Primary Network", IEEE Trans. Networking, vol. 18, no. 4, (2010) August.

[16] M. Franceschetti, O. Dousse, D. Tse and P. Thiran, "Closing The Gap In The Capacity Of Wireless Networks Via Percolation Theory”, IEEE Trans. Info. Theory, (2007) March, pp. 1009-1018.

[17] H. Zhang and Z. Zhang, "Energy Efficient Joint Source and Channel Sensing in Cognitive Radio Sensor Networks", Proceedings of 2011 IEEE International Conference on Communications (ICC), Kyoto, Japan, (2011) June 5-9.

[18] A. Ghasemi and E. S. Sousa, "Capacity of Fading Channels Under Spectrum-Sharing Constraints", IEEE International Conference on Communications, Glasgow, Scotland, UK, (2006) June, pp. 43734378 . 
[19] N. Devroye, P. Mitran and V. Tarokh, "Achievable Rates in Cognitive Radio Channels", IEEE Transactions on Information Theory, vol. 52, (2006) May, pp. 1813-1827.

[20] W. Wu, S. Vishwanath and A. Arapostathis, "On the Capacity of the Interference Channel with Degraded Message Sets", Submitted to the IEEE Transactions on Information Theory, not publication, (2006).

[21] H. Zhang and Z. Zhang, "Energy Efficient Joint Source and Channel Sensing in Cognitive Radio Sensor Networks", 2011 IEEE International Conference on Communications (ICC), Kyoto, Japan, (2011) June 5-9.

[22] H. Zhang, Z. Zhang and H. Dai, "On the Capacity Region of Cognitive Multiple Access over White Space Channels", IEEE Journal on Selected Areas in Communications, vol. 31, no. 11, (2013), pp. 2517-2527.

[23] Q. Sun, F. Hu and Q. Hao, "Context Awareness Emergence for Distributed Binary Pyroelectric Sensors", In Proceeding of IEEE International Conference of Multi-sensor Fusion and Integration of Intelligent Systems, Utah, USA, (2010) September, pp. 162-167.

[24] F. Hu, Q. Sun and Q. Hao, "Mobile Targets Region-of-Interest via Distributed Pyroelectric Sensor Network: Towards a Robust, Real-time Context Reasoning", In Proceeding of IEEE International Conference on Sensors, Da Nang, Vietnam, (2010), pp. 1832-1836.

[25] X. Huang, G. Wang and Q. Sun, "QoS Adaptive Routing Design for Multi-hop Cognitive Radio Networks Considering Multi-link Interference", International Journal of Sensors, Wireless Communications and Control, vol. 1, (2011), no. 88-92.

[26] Y. Zhou, L. Li, T. Zhao and H. Zhang, "Region-Based High-Level Semantics Extraction With CEDD", In Proceeding of 2010 2nd IEEE International Conference on Network Infrastructure and Digital Content, Hangzhou, China, (2010) October, pp. 404-408.

[27] Y. Zhou, L. Li and H. Zhang, "Adaptive Learning of Region-based pLSA Model for Total Scene Annotation", arXiv preprint arXiv:1311.5590, (2013).

[28] J. Y. Wu, W. J. Li, J. P. Huang, J. L. Zhang and D. R. Chen, "Key Techniques For Mobile Internet: A Survey", SCIENTIA SINICA Informationis, vol. 45, no. 1, (2015), pp. 45-69.

\section{Author}

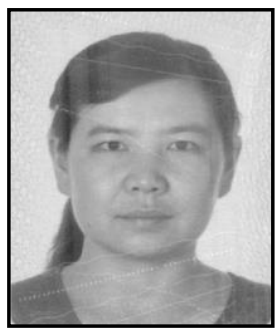

Dong Ting, was born in October 1981, Shaanxi Qishan. She got the mast degree of engineering of Sichuan University in 2011. Now she is a lecture of Information Engineering College of Yulin University since 2004. Now her research direction includes software engineering. 
International Journal of Future Generation Communication and Networking Vol. 8, No. 5 (2015) 